\title{
STARTER-ADDER METHODS IN THE CONSTRUCTION OF HOWELL DESIGNS
}

\author{
B. A. ANDERSON and K. B. GROSS
}

(Received 2 September, 1976)

Communicated by W. D. Wallis

\begin{abstract}
The powerful starter-adder theorems for constructing Howell Designs are improved and consequently many types of Howell Designs that previously could only be constructed by multiplicative techniques are shown amenable to a modified starter-adder method. The existence question for Howell Designs of many new types $H(s, 2 n)$ is settled affirmatively. For prime powers $p^{n}, p \geqq 7$, we reduce the entire existence question for designs of type $H^{*}\left(p^{n}, 2 r\right)$, $p^{n}+1 \leqq 2 r \leqq 2 p^{n}$, to the corresponding question for designs of type $H^{*}(p, 2 m), p+1 \leqq 2 m \leqq 2 p$. If these designs exist, $s$ has no prime divisors $<7$ and $t$ odd is "close" to 1 , a design $H^{*}(s, s+t)$ is shown to exist.
\end{abstract}

\section{Introduction}

Suppose $X$ is a set such that $|X|=2 n$. A Howell Design on $X$ of type $H(s, 2 n)$ consists of a square array of side $s$ such that (1) each cell is either empty or contains an unordered pair of elements taken from $X$, (2) each element of $X$. appears exactly once in each row and each column of the array and (3) every unordered pair appears at most once in a cell of the array. It is easy to see that $n \leqq s \leqq 2 n-1$. When we wish to draw attention to the set $X$ we will use the notation $H(X, s, 2 n)$. If $Y \subset X$ such that $|Y|=2 n-s$ and no pair of elements of $Y$ occupy a cell of $H(X, s, 2 n)$, we will denote this fact notationally by either $H_{Y}(X, s, 2 n)$ or $H^{*}(s, 2 n)$.

Howell Designs of type $H(2 n-1,2 n)$ are often called Room Squares. Room squares are known to exist for all $n$ except $n=2,3$. In a recent paper, Hung and Mendelsohn (1974), constructions were given for many classes of Howell Designs. These constructions were generalizations of those known for Room Squares and included both starter-adder and multiplicative methods. However, the question of existence for many types $H(s, 2 n)$ was left unsettled and starter-adder techniques for $s$ "close" to $2 n$ were not found. Mullin and 
Wallis (1975) give a condensed proof for the general existence of Room Squares. The argument is in basically two steps and proceeds roughly in the following manner. First show that if $2 n-1 \geqq 7$ is a prime power, an $H(2 n-1,2 n)$ can be constructed by the starter-adder method. Then appeal to certain multiplication theorems to complete the proof. It is clearly the premise of the above mentioned paper by Hung and Mendelsohn that the same idea might work for Howell Designs in general.

In this paper we are concerned primarily with the first step in the proposed general argument. We are able to modify the starter-adder method to show that if $p \geqq 7$ is prime and all possible $H^{*}(p, 2 m)$ exist, then for every positive integer $n$, all possible $H^{*}\left(p^{n}, 2 r\right)$ exist. Thus, one could repeat the first step of the Mullin-Wallis paper for the more general Howell Design situation if it could be shown that for prime $p \geqq 7$, all possible $H^{*}(p, 2 m)$ exist. For $p$ prime, $7 \leqq p \leqq 23$, only the $H^{*}(p, 2 p-2)$ existence question is still in doubt and a design of type $H(7,12)$ is known.

Intuitively, our modified starter-adder construction is as follows. We omit a subgroup from the starter and fill in the holes of the resulting design with a Howell Design of side the order of the subgroup.

To avoid confusion, we note that a design very similar to the ones described above is called a Howell rotation in the papers of Parker and Mood (1955), Berlekamp and Hwang (1972) and Schellenberg (1973). In those papers the array is not always required to be square, and the cells of the array are filled with ordered pairs. One also requires some other things of the design which the reader who wishes to investigate will find described therein.

A method of construction of Howell Designs that is different from those described here and also settles the existence question for some new types $H(s, 2 n)$ is found in Anderson (to appear).

\section{Generalized starters and Howell Designs}

We first establish some notational conventions. $G$ will always denote a finite Abelian group written additively. If $G$ is given and $\Lambda=\left\{F_{:}: 1 \leqq i \leqq n\right\}$ is a family of subgroups of $G$, we will use the notation $G \backslash \Lambda$ for $G \backslash \cup\left\{F_{i}: 1 \leqq i \leqq n\right\}$. If $1 \leqq i<j \leqq n$ implies that $F_{i} \cap F_{j}=\{0\}$, we will say that $\Lambda$ is a pairwise almost disjoint (PAD) family of subgroups.

Definition 1. Suppose that $G$ and $\Lambda$ are given such that $|G \backslash \Lambda|$ is even. A partition $X$ of $G \backslash \Lambda$ into (unordered) 2-sets is said to be a partial starter for $G \backslash \Lambda$ if and only if

$$
\{a-b:\{a, b\} \in X\}=G \backslash \Lambda \text {. }
$$


If $X$ is a partial starter for $G \backslash \Lambda$ and $A: X \rightarrow G \backslash \Lambda$ is an injection satisfying the condition

$$
\cup\{\{a, b\}+\{a, b\} A:\{a, b\} \in X\}=G \backslash \Lambda
$$

then $A$ is said to be a partial adder for $X$. If

$$
\{a+b:\{a, b\} \in X\}
$$

is a set of $|X|$ distinct elements of $G \backslash \Lambda$, then $X$ is said to be a strong partial starter for $G \backslash \Lambda$.

Sometimes it will be advantageous to use the notations $p S(G \backslash \Lambda)$, $p A(G \backslash \Lambda)$ and $S p S(G \backslash \Lambda)$ for the concepts defined above. Note that even if $|G \backslash \Lambda|$ is even, there still may be no $p S(G \backslash \Lambda)$. This is certainly the case if $G \backslash \Lambda$ has an element of order 2. As in the ordinary starter situation, if $X$ is a $\operatorname{SpS}(G \backslash \Lambda)$, then the map $M$ defined by $\{a, b\} M=-(a+b)$ is a $p A(G \backslash \Lambda)$ for $X$.

Before stating the first theorem we recall an idea from Hung and Mendelsohn (1974). If $U_{2} \subset U_{1}$ and $H\left(U_{2}, s_{2}, 2 n_{2}\right)=A_{2}$ is a subsquare of $H\left(U_{1}, s_{1}, 2 n_{1}\right)=A_{1}$, we will denote by $A_{1} \backslash A_{2}$ the design obtained by deleting $A_{2}$ from $A_{1}$, that is, $A_{1} \backslash A_{2}$ is the collection of cells and entries of $A_{1}$ that are not in $A_{2}$.

If $T=\left\{t_{i}: 1 \leqq i \leqq n\right\}$ is a family of non-negative integers, let $\Omega_{T}=$ $\left\{D_{i}: 1 \leqq i \leqq n\right\}$ be a family of sets such that $D_{i}=E_{i} \cup\{x\}, 1 \leqq i<j \leqq n$ implies that $E_{i} \cap E_{j}=\varnothing$ and $\left|E_{i}\right|=t_{i}$. (We will say that $\Omega_{T}$ is a PAD family of sets with common element $\infty$ ). Let $D=\cup\left\{D_{i}: 1 \leqq i \leqq n\right\}$.

THEOREM 1. Suppose $\Lambda=\left\{F_{i}: \mathrm{i} \leqq i \leqq n\right\}$ is a PAD family of subgroups of $G, G \backslash \Lambda$ has a $p S(G \backslash \Lambda)$ called $S$ and $S$ has a pA $(G \backslash \Lambda)$ called $A$. Suppose $T$ and $\Omega_{T}$ are as above and that for $1 \leqq i \leqq n$, there is an

$$
H_{D_{i}}\left(F_{i} \cup D_{i},\left|F_{i}\right|,\left|F_{i}\right|+t_{i}+1\right)=H_{i} .
$$

We may assume without loss of generality that the rows and columns of $H_{i}$ are labelled by the elements of $F_{i}$ and that for each $f$ in $F_{i},\{f, \infty\}$ occupies the cell in row fand column $f$, where $\infty$ is the common element of $\Omega_{r}$. Then there is an

$$
H_{D}\left(G \cup D,|G|,|G|+1+\sum_{i=1}^{n} t_{i}\right)=K
$$

containing each $H_{i}$ as a subsquare and such that for $1 \leqq i \leqq n, K \backslash H_{i}$ contains no cell with a pair $\{u, v\}$, where $u, v \in F_{i} \cup D_{i}$.

Proof. Label the group elements $0=g_{1}, g_{2}, \cdots, g_{G \mid}$ and label the rows and columns of a $|G| \times|G|$ array with the group elements. Let $c\left(g_{i}, g_{j}\right)$ denote the cell in row $g_{i}$ and column $g_{j}$. There are two parts to the construction. 
First, $S$ and $A$ are used as in the starter-adder construction for Room Squares.

(1) For $h \in G,\{a+h, b+h\} \in c(h, h-(\{a, b\} A))$ if and only if $\{a, b\} \in S$. The cells filled by (1) are cells whose coordinates differ by elements of $G \backslash \Lambda$. It is clear that every pair that has been used to fill a cell differs by elements of $G \backslash \Lambda$ and it is easy to see that every pair that differs by elements of $G \backslash \Lambda$ has been used exactly once. It also follows easily that the elements of $G$ that don't appear in row $g_{i}$ and column $g_{i}$ are exactly the elements $\left(U F_{i}\right)+g_{\text {. }}$.

Second, each $H_{i}$ is placed in the $F_{i} \times F_{i}$ subarray and translated by cosets. This is done as follows. For each $i, 1 \leqq i \leqq n$, let $R_{i}$ be a set of representatives of the cosets of $F_{i}$ such that $0 \in R_{i}$. If $s \in R_{i}$, let $C_{s, i}=\left\{c(f+s, g+s): f, g \in F_{i}\right\}$ and let $C_{i}=\cup\left\{C_{s, i}: s \in R_{i}\right\}$. For each $i$, if $r, s \in R_{i}$ and $r \neq s$, then $C_{s, i} \cap C_{r, i}=$ $\varnothing$ and for $i \neq j$, the fact that $\Lambda$ is a PAD family insures that

$$
C_{i} \cap C_{j} \subset\{c(g, g): g \in G\} .
$$

For each $i$, extend the group operation to $D_{i}$ by defining $d+g=g+d=d$ for all $g \in G$ and $d \in D_{i}$.

Fill in $C_{0, i}$ with $H_{i}$ and translate $H_{i}$ as follows.

(2) For $s \in R_{i}$,

$$
\{a+s, b+s\} \in c(f+s, g+s) \text { if and only if }\{a, b\} \in c(f, g) ; f, g \in F_{i} .
$$

We denote the array defined by (2) by $H_{\mathrm{s}, \mathrm{i}}$. Clearly $H_{\mathrm{s}, \mathrm{i}}$ fills the collection $C_{\mathrm{s}, \mathrm{i}}$ of cells. Note that the cells filled by the $H_{i}$ 's and their translates were all open after the first stage because these cells all have coordinates that differ by elements of $\cup F_{i}$. The assumptions made about the $H_{i}$ 's in the statement of the theorem imply that when a cell is in both $C_{i}$ and $C_{j}$, it is filled with the same pair by the proper translates of both $H_{i}$ and $H_{j}$. Every pair of elements of $G$ used in connection with $H_{i}$ differs by elements of $F_{i}$. Since cosets of the same subgroup are pairwise disjoint and since $\left\{F_{i}: 1 \leqq i \leqq n\right\}$ is a PAD family, no pair of elements of $G$ that differ by elements of $U F_{i}$ is used more than once. Each element of $D_{i}$ is paired with every element of $F_{i}$ exactly once in $H_{i}$ and thus with every element of $G$ exactly once in the translates of $H_{i}$. No pairs of elements of $D_{i}$ occur in $H_{i}$ and so none occur in the translates of $H_{i}$ either.

Consider row $g_{j}$. Given $i$, there is an $s \in R_{i}$ such that $g_{j} \in F_{i}+s$. Thus $C_{s, i}$ has cells in row $g_{i}$. Hence every element of $F_{i}+s=F_{i}+g_{i}$ and every element of $D_{i}=E_{i} \cup\{x\}$ appears exactly once in row $g_{j}$ and $\infty$ appears in $c\left(g_{i}, g_{j}\right)$. Since this argument holds for every $i$, it follows that row $g_{i}$ contains every element of

$$
\left[\left(\cup F_{i}\right)+g_{i}\right] \cup D
$$

exactly once. The same result holds for column $g_{i}$. 
Finally, suppose that for each $i$, no two elements of $D_{i}$ occupy a cell of $H_{i}$. It is clear that no two elements of $D$ occupy any cell of the entire array $H$ and it is easy to see that no pair of elements of $F_{i} \cup D_{i}$ occurs in any cell of $H \backslash H_{i}$.

The designs resulting from this theorem can be used in connection with Theorems 6 and 10 of the Hung and Mendelsohn paper. Note that the subsquare hypotheses of Theorem 10 of their paper will not be satisfied with respect to $H_{i}$ unless $\Sigma_{j \neq i} t_{j}=0$. We remark that if $\Lambda=\{0\}$, the construction reduces to the ordinary starter-adder method for Room Squares. The most interesting application of Theorem 1 occurs when $\cup F_{i}=G$.

THEOREM 2. Suppose $p \geqq 7$ is a prime and if $p+1 \leqq 2 m \leqq 2 p$, then $H^{*}(p, 2 m)$ exists. It follows that if $n$ is a positive integer and $p^{n}+1 \leqq 2 r \leqq 2 p^{n}$, then $H^{*}\left(p^{n}, 2 r\right)$ exists.

Proof. Let $Z_{p}$ be the cyclic group of order $p$ and let $G_{p, n}=Z_{p} \times \cdots \times Z_{p}$ with $n$ factors. Then $G_{p, n}$ is the union of a PAD family $\Lambda$ of $\sum_{i=1}^{n}\left(\begin{array}{l}n \\ i\end{array}\right)(p-1)^{i-1}$ subgroups of order $p$. Now, by Mullin and Nemeth (1969) and Chong and Chan (1974), $Z_{p}$ has a strong starter. We may use this strong starter on every member of $\Lambda$. It is therefore clear that if $\Gamma$ is any subset of $\Lambda$, there is a $\operatorname{SpS}(G \backslash \Gamma)$. Choose $T$ such that each $t_{i}$ is an even non-negative integer, $t_{i} \leqq p-1$ and $\sum t_{i}=2 r-\left(p^{n}+1\right)$. One may use the construction or the Binomial Theorem to conclude that

$$
(p-1)\left[\sum_{i=1}^{n}\left(\begin{array}{c}
n \\
i
\end{array}\right)(p-1)^{i-1}\right]+1=p^{n}
$$

and the result follows by Theorem 1 .

It seems likely that given $p \geqq 7$, most of the $H^{*}(p, 2 m)$ exist. Note that if all $H^{*}(p, 2 m)$ exist except possibly $H^{*}(p, 2 p-2), p \geqq 7$, then the method of Theorem 2 will show that all $H^{*}\left(p^{n}, 2 r\right)$ exist except possibly $H^{*}\left(p^{n}, 2 p^{n}-2\right)$. Some of the currently available information on this question was mentioned in the introduction. Note also tht the proof of Theorem 2 gives an easy way of extending strong starters from $Z_{p}$ to $G_{p, n}$.

The preceding results have all assumed the existence of a PAD family of subgroups. We now show that in certain situations this requirement can be relaxed.

Definition 2. Suppose $G$ is a finite Abelian group and $X$ is a partition of $G$ into singletons $S_{X}$ and doubletons $D_{X}$. We will say that $X$ is an $H M$-starter if and only if

1) $\{a, b\} \in D_{x}$ implies $a-b \neq b-a$,

and 
2) $\{a, b\},\{c, d\}$ distinct elements of $D_{x}$ implies $a-b \neq \pm(c-d)$.

If $A: X=S_{X} \cup D_{X} \rightarrow G$ is an injection satisfying the condition

$$
\left(\cup\left\{s+\{s\} A:\{s\} \in S_{X}\right\}\right) \cup\left(\cup\left\{\{a, b\}+\{a, b\} A:\{a, b\} \in D_{X}\right\}\right)=G
$$

then $A$ is said to be an $H M$-adder for $X$.

It is easy to see that if $X$ is an $H M$-starter for $G$, then $S_{X} \neq \varnothing$. Note that this is true whether $|G|$ is odd or even. Thus by simple translation, we may assume that $\{0\} \in S_{X}$ and that if $A$ is an $H M$-adder for $X$, then $\{0\} A=0$.

The next result was mentioned in Hung and Mendelsohn (1974) for cyclic groups only, but is true in the more general setting.

Theorem 3. Suppose $G$ is an Abelian group with HM-starter $X$ and $H M$-adder $A$ for $X$. If $D$ is a set of $\left|S_{X}\right|$ ideal elements, then there is an $H_{D}\left(G \cup D,|G|,|G|+\left|S_{X}\right|\right)$.

Definition 3. Suppose $G$ is a finite Abelian group, $\Lambda=\left\{F_{i}: 1 \leqq i \leqq n\right\}$ is a family of subgroups of $G, X$ is a partition of $\cup F_{i}$ into singletons and doubletons and $A: X \rightarrow \cup F_{\mathrm{i}}$ is an injection. We will say that $(X, A)$ is an $H M$-partition of $\Lambda$ if and only if

1) each $F_{i}$ is a union of partition elements of $X$, and

2) for each $i, 1 \leqq i \leqq n, X \mid F_{i}$ is an $H M$-starter and $A \mid\left[X \mid F_{i}\right]$ is an $H M$-adder for $X \mid F_{i}$.

The next result is immediate.

TheOREM 4. Suppose $G$ is an Abelian group and $\Lambda=\left\{F_{i}: 1 \leqq i \leqq n\right\}$ is a family of subgroups of $G$. Suppose $S$ is a partial starter for $G \backslash \Lambda$ and $B$ is $a$ partial adder for $S$. If $(X, A)$ is an $H M$-partition of $\Lambda$, then $X \cup S$ is an $H M$-starter for $G$ and the function $C$ such that $C \mid \Lambda=A$ and $C \mid G \backslash \Lambda=B$ is an $H M$-adder for $X \cup S$.

The existence of $H M$-partitions may be an interesting question for consideration. For now, we content ourselves with the following observation.

Theorem 5. Suppose $G$ is an Abelian group and $\Lambda=\left\{F_{1}: 1 \leqq i \leqq n\right\}$ is $a$ family of subgroups all of odd order. If $X$ is a partial starter for $G \backslash \Lambda, A$ is $a$ partial adder for $X$ and $D$ is a family of ideal elements such that $|D|=\left|\cup F_{i}\right|$, then there is an

$$
H_{D}\left(G \cup D,|G|,|G|+\left|\cup F_{i}\right|\right) \text {. }
$$

Proof. An obvious extension of Theorem 2 of Hung and Mendelsohn (1974) allows one to conclude that for each $i, S_{i}=\left\{\{g\}: g \in F_{i}\right\}$ is an $H M$-starter for $F_{i}$ and the map $\{g\} \rightarrow g$ is an $H M$-adder $A_{t}$ for $S_{i}$. In this way 
we construct an $H M$-partition of $\Lambda$ and the result follows from Theorems 3 and 4 .

\section{Existence of strong partial starters}

The utility of Theorem 1 is clearly affected by the availability of partial starters and partial adders. In this section, it will be shown that they can often be constructed. Even though most of these results could be stated in terms of partial starters and partial adders, we will avoid the additional complexity this sometimes introduces and consider only stong partial starters. We first state the obvious

THEOREM 6. If $G$ has subgroups $L, T$ with $L \subset T$ and there is a $S p S(G \backslash T)$ and a $\operatorname{SpS}(T \backslash L)$, then there is a $\operatorname{SpS}(G \backslash L)$.

Definition 4. Suppose $F$ is a finite Abelian group with identity permutation $I$. A permutation $Y$ of $F$ is said to be a strong orthomorphism of $F$ if and only if both $Y+I$ and $Y-I$ are permutations of $F$.

An important result for certain doubling constructions that we hope to consider in a later paper is that if $F=G_{2, n}, n>1$, then $F$ has a strong orthomorphism, Paige (1947). It is also known that if $G$ has a cyclic 2-Sylow subgroup, then $G$ does not have a strong orthomorphism, Hall and Paige (1955), and if $G$ has odd order divisible by 3 with a cyclic 3-Sylow subgroup, $G$ does not have a strong orthomorphism, Gross and Leonard (1975).

THEOREM 7. If there is a strong orthomorphism for $H$ and a strong orthomorphism for $G / H$, then there is a strong orthomorphism for $G$.

Proof. Let $C$ be a function that selects coset representatives for $G / H$. Let $Y_{H}$ and $Y_{G / H}$ be strong orthomorphisms for $H$ and $G / H$ respectively. Define a map $Y$ by the rule

$$
(a C+h) Y=\left(a Y_{G / H}\right) C+h Y_{H} ; a \in G / H \text { and } h \in H .
$$

It is straightforward to verify that $Y$ has the required properties.

It turns out that strong orthomorphisms allow the construction of many strong partial starters as in

THEOREM 8. If there is a $\operatorname{SpS}(G / H \backslash N / H)$ and a strong orthomorphism of $H$, then there is a $\operatorname{Sp} S(G \backslash N)$.

Proof. Let $S$ be a $S p S(G / H \backslash N / H)$, let $Y$ be a strong orthomorphism of $H$ and let $C$ be a function that selects coset representatives of $G / H$. Then the following partition is an $\operatorname{Sp} S(G \backslash N)$.

$$
\{\{a C+h, b C+h Y\}:\{a, b\} \in S, h \in H\} .
$$


In many cases this result can be applied to build a strong partial starters from strong starters.

THEOREM 9. If $|G|$ is not divisible by 2,3 or 5 , and $H$ is a subgroup of $G$, then there is an $\operatorname{SpS}(G \backslash H)$.

Proof. There is a $\operatorname{SpS}(G / H \backslash H / H)$ by the results of Gross and Leonard (1975 and 1976) and since $3 \not|H|$, there is a strong orthomorphism of $H$, say multiplication by 2 . By Theorem 8 , there is a $\operatorname{SpS}(G \backslash H)$.

It is possible to generalize Theorem 9 to certain PAD families of subgroups.

Theorem 10. Suppose $G$ is Abelian and $\Lambda=\left\{F_{i}: 1 \leqq i \leqq n\right\}$ is a PAD family of subgroups of $G$ such that the group $\langle\Lambda\rangle$ generated by the $F_{i}$ 's is isomorphic to $\times\left\{F_{1}: 1 \leqq i \leqq n\right\}$. If $G$ is not divisible by 2,3 or 5 , then there is a $\operatorname{SpS}(G \backslash \Lambda)$.

Proof. First, $G \backslash\langle\Lambda\rangle$ has a $S p S$ by Theorem 9. We will let $N=$ $\{i: 1 \leqq i \leqq n\}$ and say that if $f=\left(f_{1}, f_{2}, \cdots, f_{n}\right) \in \times F_{i}$ and $f_{i} \neq 0$ if and only if $i \in M \subset N$, then $f$ is of type $M$ and basic type $|M|$. Multiplication by 2 is a strong orthomorphism $Y$ of $X F_{i}$ that preserves type.

In order to prove the theorem, it will suffice to define a strong starter on $\times F_{i}$ that pairs points of the same type and has the property that every sum of a pair of the starter is of the same type as the members of the pair.

Each $F_{\mathrm{i}}$ has a strong starter $S_{\mathrm{i}}$. Suppose all points of basis type $r$ have been paired with points of the same type such that sums of pairs preserve type. Let $R^{+}=R \cup\{i\} \subset N$ be a set of basic type $(r+1)$ such that $R$ is of basic type $r$. Let $H=\times\left\{F_{j}: j \in R\right\}$ and $K=H \times F_{i}$. We know that $Y \mid H=Y_{H}$ is a strong orthomorphism of $H$. Let

$$
S_{K}=S_{H} \cup\left\{\left\{\left\{h+s_{1}, h Y_{H}+s_{2}\right\}: h \in H\right\}:\left\{s_{1}, s_{2}\right\} \in S_{i}\right\} .
$$

By Gross and Leonard (1975) or Anderson and Morse (1974), this is a strong starter on $K$. Furthermore, it is clear that $S_{K}$ pairs points of type $R^{+}$and such pairs sum to an element of type $R^{+}$. Add the pairs of $S_{K}$ that are both of type $R^{+}$to the pairs of basic type $r$ that we already have. Since $R^{+}$was an arbitrary subset of $N$ of basic type $r+1$, we see that a strong starter with the required properties can be defined on $\times F_{i}$.

-THEOREM 11. Suppose $n=\Pi_{j=1}^{m} p_{j}^{i_{j}}$ is the factorization of $n$ into prime powers such that $r<s$ implies $p_{r}<p_{s}$. Suppose further that $7 \leqq p_{1}$ and that for all $\mathrm{j}$, if $p_{j}+1 \leqq 2 m \leqq 2 p_{i}$, then $H^{*}\left(p_{j}, 2 m\right)$ exists. It follows that for $t$ odd, $1 \leqq t \leqq 1+\sum_{i=1}^{m}\left(p_{i}^{i}-1\right)$, there is an $H^{*}(n, n+t)$. 
Proof. For each $j$, let $G_{j}$ be the product of $i_{j}$ cyclic groups of order $p_{j}$ and let $G=\times\left\{G_{i}: 1 \leqq j \leqq m\right\}$. For each $k, j, 1 \leqq k, j \leqq m$, let $\delta_{k j} G_{j}$ be $\{0\}$ if $k \neq j$ and $G_{j}$ if $k=j$. Then for each $j$, let $F_{j}=\times\left\{\delta_{k j} G_{j}: 1 \leqq k \leqq m\right\}$. Clearly $\Lambda=\left\{F_{;}: 1 \leqq j \leqq m\right\}$ is a PAD family of subgroups of $G$ that satisfies the hypothesis of Theorem 10 . Therefore, there is a $\operatorname{SpS}(G \backslash \Lambda)$. The result now follows by applying Theorems 1 and 2 .

As in the case of Theorem 2, note that even if all the $H^{*}\left(p_{i}, 2 m\right)$ don't exist, this result yields many Howell Designs. We conclude with a construction that can be useful if 2,3 or 5 divide the order of $G$.

TheOREM 12. Let $N, H, U$ and $G$ be Abelian groups such that $N \subset H \subset$ $U \subset G$. Suppose that there is a $\operatorname{SpS}(G / H \backslash U / H)$ and $U / H \cong N$. Suppose also that $(|H / N|,|N|)=1, H / N$ has a strong orthomorphism and there is a $S p S(H \backslash N)$. Then for $V \subset U$ such that $V / N \cong N$, there is a $S p S(G / N \backslash V / N)$.

Proof. Since $G / H \cong(G / N) /(H / N)$ and $U / H \cong(U / N) /(H / N)$, it follows from Theorem 8 that there exists a $S p S(G / N \backslash U / N)$. Now $N \cong$ $(U / N) /(H / N)$ and $(|H / N|,|N|)=1$ so that by some of the basic properties of Abelian groups, $U / N \cong H$. An application of Theorem 6 completes the proof.

There are other methods of generating strong partial starters that sometimes work when the techniques mentioned here are not available. It is planned to discuss some of these methods at a later time.

\section{References}

B. A. Anderson (to appear), 'Howell designs of type $H(p-1, p+1)$ '.

B. A. Anderson and D. Morse (1974), 'Some observations on starters', Proc. 5th Southeastern Conf. on Comb., Graph Theory and Computing, Winnipeg, 229-235.

E. R. Berlekamp and F. K. Hwang (1972), 'Constructions for balanced Howell rotations for bridge tournaments', J. Combinatorial Theory, Ser. A 12, 159-166.

B. C. Chong and K. M. Chan (1974), 'On the existence of normalized Room squares', Nanta Math 7, 8-17.

K. B. Gross and P. A. Leonard (1975), 'The existence of strong starters in cyclic groups', Utilitas Math. 7, 187-195.

K. B. Gross and P. A. Leonard (1976), 'Adders for the patterned starter in nonabelian groups', J. Austral. Math. Soc., Ser. A 21, 185-193.

M. Hall and L. J. Paige (1955), 'Complete mappings of finite groups', Pacific J. Math. 5, 541-549.

S. H. Y. Hung and N. S. Mendelsohn (1974), 'On Howell designs', J. Combinatorial Theory Ser. A 16, $174=198$.

R. C. Mullin and E. Nemeth (1969), 'An existence theorem for Room squares', Canad. Math. Bull. 12, 493-497.

R. C. Mullin and W. D. Wallis (1975), 'The existence of Room squares', Aequationes Math 13, $1-7$.

L. J. Paige (1947), 'A note on finite abelian groups', Bull. Amer. Math. Soc. 53, 590-593. 
E. T. Parker and A. N. Mood (1955), 'Some balanced Howell rotations for duplicate bridge sessions', Amer. Math. Monthly 62, 714-716.

P. J. Schellenberg (1973), 'On balanced Room squares and complete balanced Howell rotations', Aequationes Math. 9, 75-90.

Arizona State University,

Tempe, Arizona 85281.

Michigan State University,

East Lansing, Michigan 48824. 